ISSN 1112-9867

http://www.jfas.info

\title{
DESIGNING SOUR TASTE SIGNAL MICRO-CONTROLLER SIMULATOR
}

\author{
B. Haghighi ${ }^{1}$, H. Haji-Ghasemi ${ }^{2}$ and N. Haghighi ${ }^{3, *}$ \\ ${ }^{1}$ Dept. Of Physics, Faculty of Science, Islamic Azad University, Mashhad Branch, Mashhad, \\ Iran \\ ${ }^{2}$ Dept. Of Physics, Faculty of Science, Islamic Azad University, Mashhad Branch, Mashhad, \\ Iran \\ ${ }^{3}$ Faculty Of Science, Islamic Azad University, Qum Branch, Qum, Iran
}

Published online: 05 June 2016

\begin{abstract}
In this paper, empirical studies on taste cells and the results of potential changes related to sour taste are used, and by studying the messages transmitted by the nervous system and equivalent sample signal of this taste, mathematical equations related to these changes are obtained, and by designing a microcontroller circuit capable of creating changes in terms of potential over time, we will be able to place the sour taste simulator microcontroller circuit in the taste cells instead of physical matter so that the same feeling become transmitted to the brain.
\end{abstract}

Keywords: Spontaneous signals; Simulated-Microcontroller.

Author Correspondence, e-mail: haghighi_236@mail.ru

doi: http://dx.doi.org/10.4314/jfas.8vi2s.57

\section{INTRODUCTION}

Sensation of taste originates from a group of cells called taste buds, which report sensation of taste to some centers in the brain stem. Sensation of taste is equivalent to stimulation of taste receptors, and these receptors are known for a large number of chemicals, contributing to perception of taste. When the stimulant enters into the oral cavity, they may bind to taste 
receptor cell membranes, pass through certain channels or activate ion channels. Feeling sour taste is actually related to the accumulation of hydrogen ions. Taste receptor cells interact with entering chemicals to create electrical signals. Once taste receptor cells are stimulated by interacting with chemicals, they become nonpolar, and this no polarity leads to actual potential, transferred to the taste nerve fibers, and eventually reaches the brain [1]. Electroencephalograph (EEG) is the recording of brain electrical activity, including picking signals from surface electrodes, improving the signals (often amplification and noise reduction), printing the signal and analyzing it. In this paper, a summary of the nervous system action and how message are transferred and then recording and analyzing signals related to the sense of taste will be studied. Here we examine the diagrams obtained from direct results of the taste cells of tongue that are in contact with the sour material, and ignore reviewing EEG when tasting sour because of high error.

\section{NERVOUS SYSTEM AND MESSAGES TRANSMISSION USING POTENTIAL DIFFERENCE}

Nervous system and the endocrine system have the responsibility of control and communication between organs and tissues of the body. Active part of the nervous system consists of nerve cells or neurons. In order to perform messaging tasks, neurons must receive information from other neurons to create nerve signals after adjustment and processing. Afterwards, the generated nerve impulse must be sent to the regions and areas associated with communication field of neuron.

Neurons have membrane that separates the contents of the cell membrane from its external environment. There are ion charge differences in capacity of the cell membranes, particularly neurons, which keeps the inner surface of the cell membrane in negative potentialstate compared to the membrane potential itself (up to several tens of millivolts). The potential of the cell's outer surface is considered as ground potential. The roles and signaling functions of the nervous system mainly occurs through potential changes in the membranes of neurons. Neurons communicate with each other in regions called synapses. All living cells have electrical potential difference on both sides of their membrane, so that inside of the membrane is negative relative to outside of the membrane. In resting neurons and muscle cells, the potential difference is several tens of $\mathrm{mV}$. Potential difference is due to the unequal distribution of ions on both sides of the cell membrane. The unequal distribution of ions is 
established and maintained by the metabolic activity of cells.

\section{RECORDING TASTE CELLS POTENTIAL}

Taste receptor cells in taste buds are able to produce action potentials in response to taste stimuli. Results showed that taste buds release spontaneous signals simultaneously and give different responses to different taste stimuli. Therefore, using taste cells as sensitive materials to create a taste sensor is an independent trend in research and development of an electronic language that uses bio molecular function units to create highly sensitive sensors. Taste receptor cells respond to stimuli was coded for the first time in itillium taste buds action potentials. The first stage of coding is a specific stimulus interaction with receptors or ion channels in taste receptor cells, which triggers a complex series of events following taste stimuli that leads to creating taste potential in receptor cells [2].

Cellular networks taste bud cell and contain all kinds of taste receptor cells. Therefore, taste buds play a very important role in the sense and transfer of taste. Recently, molecular studies have shown that individual taste attributes encoded by a variety of taste receptor cells that can discriminate the taste at cell level. Evidence Show that in sour sensations, proton $\left(\mathrm{H}^{+}\right)$can block or disable ion channels that allow other positively charged ions to enter into the cell causing its depolarization and releasing neurotransmitters [3].

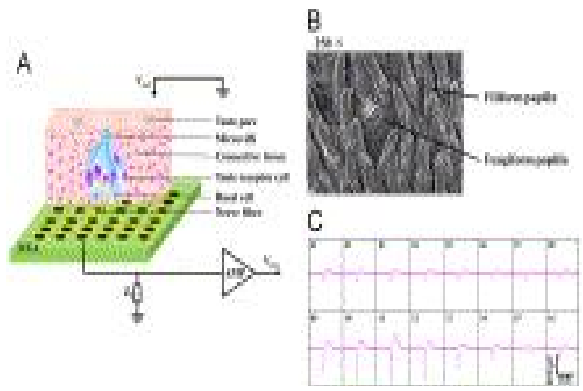

Fig.1. Extracellular potential of taste buds recorded by a microelectrode

(A) Sensor's brief diagram (B) Mushroom-shaped epithelium taste observed by electron microscopy (C) Electrophysiological multi-channel signals of epithelium Microelectrode array (MEA) can register extracellular electrical activity of excitable grown cells or tissues from multiple sites in the long and non-pervasive time. When tissue is connected to the electrode, a conductive gap between the tissue and the electrode that is filled with electrolyte is created. A small piece of tissue is composed of many taste-receptor cells of which potential electrophysiological activity can be recorded in the form of the potential passing through membrane. 
Electrical signals on the surface between electrode and electrolyte are attenuated by a voltage divider through permeability of electrode and permeability of amplifier system. According to this impedance reduction of electrode and improved tissue adhesion, potential passing through membrane is eventually recorded by MEA as extracellular transductive potential. After recording taste control signals from the taste buds, five basic taste stimuli are applied to taste epithelium fixed on multi-channel MEA chip, which is suitable for recorded information mechanisms in parallel. In this comparison, the action potentials of taste receptor cells in taste buds are analyzed to the original tastes (Figure 1). In similar results, it can be seen that response to sour taste is completely different from responses to other tastes [4].

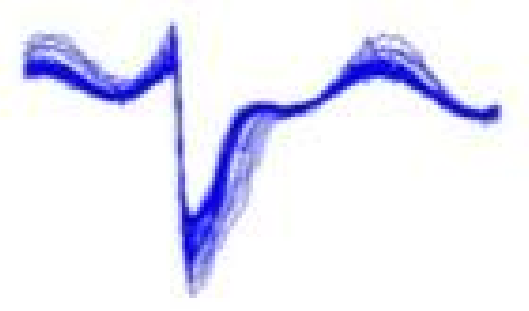

Fig.2. Changes in sour taste signal

\section{OBTAINING EQUATIONS OF SOUR TASTE SIGNAL}

By using recorded signal samples associated with sour taste (Figure 2), we extracted data signals using Getdata software.This way data are converted to digital form, and we obtain point to point changes.

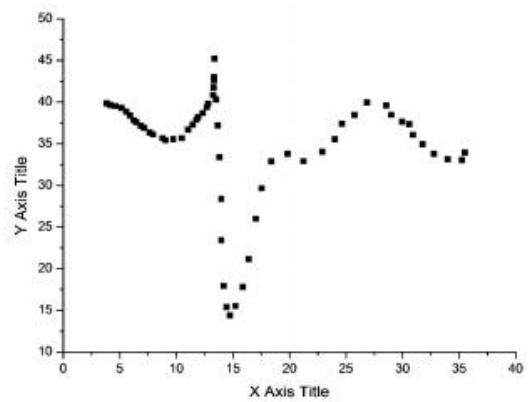

Fig.3. Charting the data after obtaining information

Using the data obtained, we draw a graph of the signal (Fig. 3). We divided the chart into three parts and obtain the function of each section by Origin software. To increase accuracy, we divide the above 
signal into three intervals and obtain the equation of each section by the software. First interval (4- 14) and for the second interval.

Diagram if Section 1, with the following function

$$
Y=54.8815-4.5037 X+0.26446 X^{2}
$$

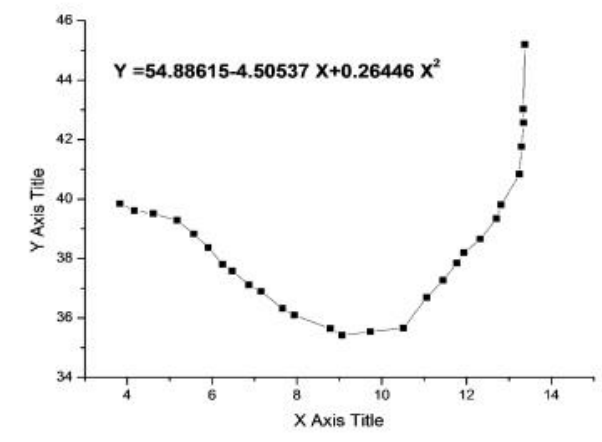

Fig.4. The diagram of the first section in 4-14 range

Diagram Section 2: its function is a Lorentz Function with the following equation:

$$
y=37.94179+\frac{28(-78.64561)}{\pi} \frac{(1.71188)}{4(x-15.4135)^{2}+(1.71188)^{2}}
$$

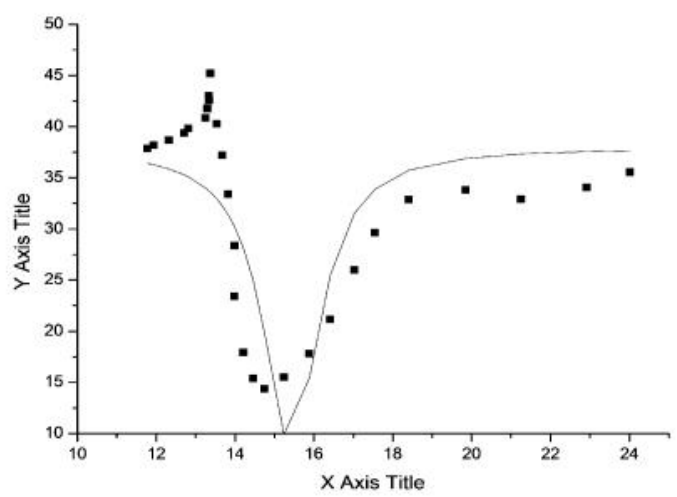

Fig.5. Diagram section 2 in range

Diagram Section 3:

$$
y=33.108424+\frac{44.90684}{5.17451 \sqrt{\frac{\pi}{2}}} e^{\frac{-2(x-27.57322) 2}{5.17451^{2}}}
$$

It has a Gaussian Function with the following equation 


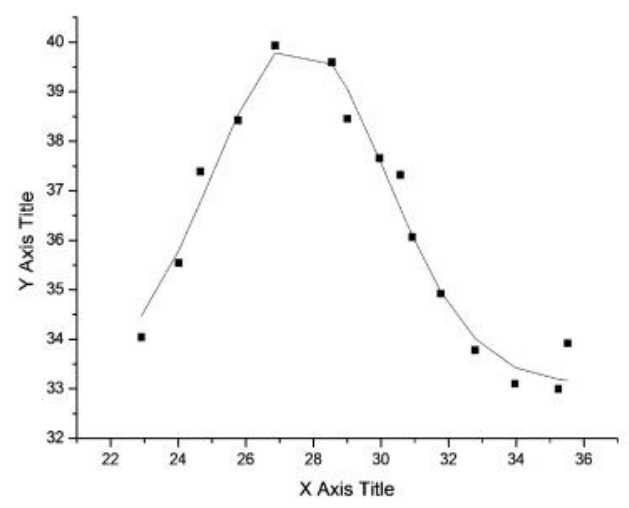

Fig.6. Diagram of section 3 of the range

Finally, the function of the signal can be obtained as follows.

$$
\begin{aligned}
& y=54.88-4.50 x+0.26 x^{2}+37.94 \\
& +\frac{2 *(-78.64)}{\pi} \frac{1.72}{4(x-15.41)^{2}+1.72^{2}}+33.11 \\
& +\frac{44.91}{5.17 \sqrt{\frac{\pi}{2}}} e^{\frac{-2(x-27.57)^{2}}{5.17^{2}}}
\end{aligned}
$$

For comparison, we draw the primary data and obtained equation on a graph as follows:

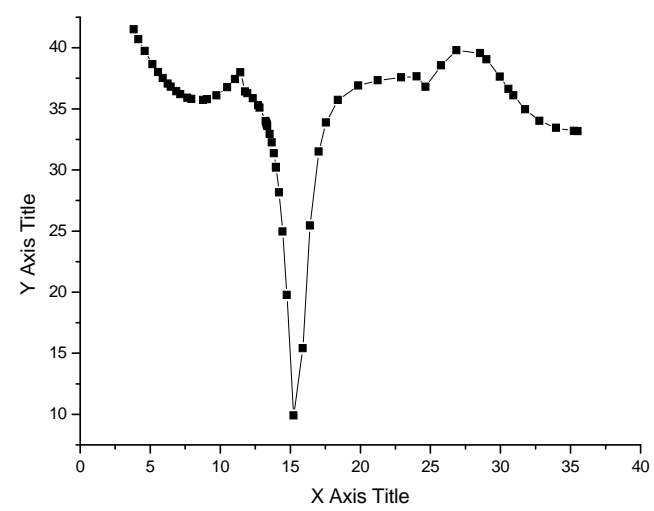

The dotted graph is the initial equation, and the line graph is the obtained graph, as it can be seen, the two graphs are identical.

\section{MICROCONTROLLER CIRCUIT SIMULATION}

We use Proteus software for circuit simulation. The software has two parts of isis and ares. Isis 
environment is used to draw and test the circuit, and ares environment is used to make the pcb of the tested circuit in isis. In brief, we can say that in Proteus, circuit simulation includes the following steps:

1. Choosing a part from library and taking it to the schematic environment

2. Placing the parts and wiring them

3. Making changes in characteristics of the parts

The circuit we intend to simulate is made of a microcontroller, a digital to analog converter, a voltmeter and a DC voltage source (Fig. 3). The first step of simulation is to put the pieces in the right place. In the next step, we specify the hex code of circuit and the frequency of the circuit, and then we start circuit simulation. The designed circuit is able to show both diagram data and diagram voltage. The circuit can be designed and implemented on an IC, and the IC can be placed on nerve cells in the tongue so that the nerve cells send the simulated signals to the brain and the brain can receive a taste similar to sour taste.

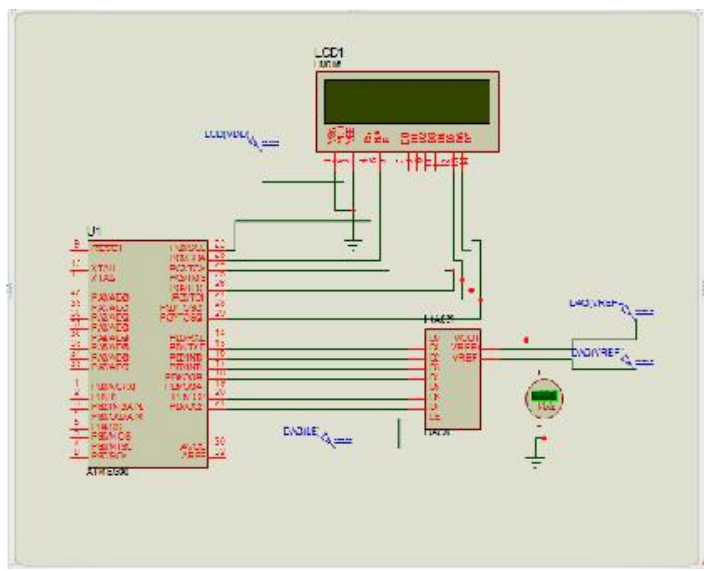

Fig.7. Circuit schematic

\section{CONCLUSION}

Studying the recorded signals associated with sense of taste, we simulate the signal using mathematical methods and simulation software and by calculating mathematical equations associated with the signal. Then, using the electronic software, we simulated an electronic device that could generate signal potential changes associated to the equation. In practice, by making this piece and placing it in nerve cells of taste buds, similar sense of sourness can be perceived in the neurons as well 
as the brain. Also, similar potential changes over time can be created. If these changes are attached to neurons of the taste cells, similar to the provided method, the sense of sour taste is induced in the nervous system.

\section{REFERENCES}

[1] Richard, Bowen - Hyper txtesfor biomedical science. Published permission, 2, 2011.

[2] El-Yassimi, A., Hichami, A., Besnard, P., Khan, NA. Journalof Biological Chemistry, 2008, 283, 12949-12959, 2008.

[3] Ishimaru, Y., Inada, H., Kubota, M., Zhuang, H., Tominaga, M., Matsunami, H. Proceedings of the National Academy of Sciences of the United States of America, 2006, $103(33), 12569-12574$.

[4] Chandrashekar, J., Hoon, MA, Ryba, NJ, Zuker, CS .Nature 444, 2006, 288-294.

\section{How to cite this article:}

Haghighi B, Haji-Ghasemi H, and Haghighi N. Designing sour taste signal micro-controller simulator. J. Fundam. Appl. Sci., 2016, 8(2S), 422-429. 